Meta

Journal des traducteurs

Translators' Journal

\title{
Le labyrinthe de " computer »
}

\section{Alain Morissette}

Volume 19, numéro 2, juin 1974

URI : https://id.erudit.org/iderudit/003668ar

DOI : https://doi.org/10.7202/003668ar

Aller au sommaire du numéro

Éditeur(s)

Les Presses de l'Université de Montréal

ISSN

0026-0452 (imprimé)

1492-1421 (numérique)

Découvrir la revue

Citer cet article

Morissette, A. (1974). Le labyrinthe de « computer ». Meta, 19(2), 87-90.

https://doi.org/10.7202/003668ar d'utilisation que vous pouvez consulter en ligne.

https://apropos.erudit.org/fr/usagers/politique-dutilisation/ 


\section{LE LABYRINTHE DU «COMPUTER»}

L'informatique, science du traitement de l'information (information processing), a fait naître une terminologie toute nouvelle qui s'implante de plus en plus. Mais comme dans toute science qui n'en est qu'à ses premières armes, le vocabulaire de l'informatique est loin d'être fixé de façon immuable. Les problèmes de traduction sont très nombreux, et les progrès accomplis dans le domaine du traitement des données ne font souvent qu'accentuer ces difficultés. Il en est ainsi du computer et du calculator rendus indifféremment dans bien des cas par calculateur, calculatrice, calculateur électronique ou ordinateur. L'imagination du traducteur y trouve son compte.

Mais voyons, en premier lieu, comment les dictionnaires anglais conçoivent le computer. A Dictionary of Computers d'Anthony Chandor donne la définition suivante : «Any machine which can accept data in a prescribed form, process the data and supply the results of the processing in a specified format $\gg$ (p. 82). 
Charles Sippl dans Computer Dictionary fournit une définition dans des termes similaires, en y ajoutant toutefois d'autres éléments : " $A$ device capable of accepting information, applying prescribed processes to the information, and supplying the results of these processes. It usually consists of input and output devices, storage, arithmetic and output devices, and a control unit $\gg(p .44)$.

Il convient de remarquer les trois éléments communs à ces deux énoncés : $A$ computer is a device capable of 1) accepting data ;2) processing the data;3) supplying the results of the processing.

Pour être en mesure d'accomplir ces trois actions fondamentales, le computer doit posséder les organes suivants : 1) input devices (input units), to accept the data; 2) arithmetic, logical, and control devices (units), to process the data; 3 ) output devices (output units), to supply the results of the processing.

Toutefois, les deux définitions citées plus haut sont incomplètes dans la mesure où le computer peut accomplir une autre opération à laquelle Sippl n'a fait qu'allusion sans l'expliquer davantage. Le Standard Dictionary of Computers and Information Processing de Weik apporte cette précision supplémentaire : « $A$ computer should be able to accept, store, execute [...] its own program» (p. 80). Le computer n'est donc pas seulement capable de recevoir des données, de les traiter et de fournir les résultats. Il peut également emmagasiner ces données ainsi que le programme en mémoire.

Ce n'est toutefois pas la mémoire en elle-même qui permet de distinguer le computer du calculator, car ces deux machines sont dotées d'éléments de mémoire, de même que l'ordinateur, le calculateur et la calculatrice en français. L'ouvrage intitulé Traité pratique d'informatique, tiré des Techniques de l'ingénieur, définit ainsi la calculatrice : «Machine de traitement de l'information spécialement adaptée à l'exécution d'opérations arithmétiques» (H540 - 4). Même cette machine qui n'effectue que des opérations élémentaires est dotée d'un type de mémoire capable de recevoir les données à additionner, à retrancher, à multiplier ou à diviser et d'une mémoire où s'effectue l'opération donnée. En français comme en anglais, toutes ces machines ont une mémoire plus ou moins perfectionnée suivant leur complexité et les opérations qu'elles doivent effectuer.

La distinction entre le computer et le calculator ne se situe donc pas au niveau de l'absence ou de la présence de la mémoire, mais provient de l'élément d'autonomie du premier, comme l'explique d'ailleurs Weik dans sa définition du calculator: « $A$ device that performs arithmetic and some limited logic operations and displays the results, but that usually cannot itself alter its own program》 (p. 44). Sous la rubrique computer, il précise : «If the program is inserted manually, the term calculator is used... A computer should be able to modify its own program... A person is no longer implied by the term $\gg$ (p. 80).

Le Traité pratique d'informatique des Techniques de l'ingénieur (H540-4), l'Organisation internationale de normalisation (ISO) dans le Vocabulaire du traitement de l'information (p. 21) de même que l'Association française de normalisation (AFNOR) dans Traitement de linformation-Vocabulaire (p. 8) reprennent la 
même distinction en français. La calculatrice est une «machine de traitement de l'information [...] dont l'emploi exige l'intervention fréquente d'un opérateur humain ». Le calculateur est une «machine de traitement de l'information susceptible d'effectuer les calculs importants comprenant de nombreuses opérations arithmétiques ou opérations logiques, sans intervention d'un opérateur humain au cours de l'exécution des calculs».

Le recoupement de ces énoncés nous permet d'établir les équivalences suivantes $:$ calculator $=$ calculatrice computer $=$ calculateur. Mais alors, par quel terme anglais peut-on rendre ordinateur? Après avoir défini le computer, Weik nous fournit la réponse : «In information processing, usually an automatic storedprogram computer is implied 》 (p. 79). Le Glossary of Terms in Computers and Data Processing de Berkeley distingue très nettement le computer du storedprogram computer : «Stored-program computer. A computer in which the instructions which specify the program to be performed are stored in the memory of the computer along with the data to be operated upon... Contrasted with a computer whose program is external 》 (p. 80).

Dans sa définition de l'ordinateur, l'AFNOR fait la même distinction en français : "Calculateur, commandé par des instructions enregistrées dans une mémoire interne, qui peut synthétiser et mettre en mémoire des instructions et les exécuter ensuite» (p. 9). Il convient de remarquer que l'AFNOR et l'ISO donnent aussi comme équivalent d'ordinateur, calculateur à programme enregistré. Du point de vue strictement terminologique, ordinateur et calculateur à programme enregistré sont donc les équivalents exacts de stored-program computer, même s'il arrive souvent que dans les textes de vulgarisation, computer est employé au sens de stored-program computer.

L'ordinateur est donc une machine dotée :

1) d'organes d'entrée (input units) pour recevoir les données et le programme qui sont lus par différents supports (mediums) : cartes perforées (punched cards), handes perforées (punched tapes), bandes magnétiques (magnetic tapes), disques magnétiques (magnetic disks) ;

2) d'une unité logique (logical unit) et d'une unité arithmétique (arithmetic unit) pour effectuer les calculs ;

3) d'une mémoire (memory, storage, store) pour emmagasiner les données et le programme ;

4) d'un pupitre de commande ou pupitre central (console) pour vérifier l'exécution des calculs;

5) d'organes de sortie (output units) pour fournir les résultats.

Comme l'affirme Weik : "Without any one of these five main units, the system is not a computer (stored-program computer) in the full sense» (p. 80). 
Nous pouvons donc résumer ainsi :

stored-program computer

computer (textes vulgarisés)

computer

calculator ordinateur, ou calculateur à programme enregistré (dans la mémoire interne)

calculateur (programme externe)

calculatrice

Alain Morissette

\section{BIBLIOGRAPHIE}

Association française de normalisation (AFNOR), Traitement de linformation. Vocabulaire, chapitre 01. Termes fondamentaux, NF Z 61-001, juin 1970.

Berkeley, Edmund C. et Linda L. Lovett, Glossary of Terms in Computers and Data Processing, $5^{e}$ éd., Newtonville (Mass.), Berkeley Enterprises, Inc., 1960, 90 p.

Chandor, Anthony, John Graham et Robin Williamson, Dictionary of Computers, Harmondsworth, Penguin Books, 1970, 407 p.

International Organization for Standardization/Organisation internationale de normalisation (ISO), Vocabulaire du traitement de l'information, chapitre 01. Termes fondamentaux.

Sippl, Charles J., Computer Dictionary, Indianapolis (Indiana), Howard W. Sams \& Co. et The Bobbs-Merril Co., 1970, 336 p.

Traité p"atique d'informatique, Paris, Techniques de l'ingénieur. $\mathrm{H} 1,1970$.

Weik, Martin H., Standard Dictionary of Computers and Information Processing, New York, Hayden Book Co., 1970, IX-326 p. 